\title{
Biomatériaux : traitements de surface par dépôt de films minces
}

\author{
Catherine Picart \\ Laboratoire de dynamique moléculaire des interactions membranaires, \\ CNRS UMR 5539, Université de Montpellier 2
}

\begin{abstract}
Un biomatériau doit être conçu non seulement pour ses propriétés de volume, mais également pour ses propriétés de surface, celle-ci constituant l'interface avec le milieu biologique environnant et jouant un rôle primordial dans de nombreux domaines (implantologies osseuse ou vasculaire, protection anti-microbienne). Traiter une surface dans le but de lui donner des propriétés spécifiques vis-à-vis de tissus et fluides biologiques représente donc un enjeu important. Dans cet article, les récents développements d'une nouvelle technique de dépôt de films minces, couche par couche, sont présentés dans le cadre d'applications au domaine des biomatériaux.
\end{abstract}

Les biomatériaux sont nés du désir de concevoir des matériaux qui s'intègrent au vivant avec succès, sans rejet ni réaction indésirable. La définition consensuelle du terme biomatériau a été donnée lors de la Conférence de la Société Européenne des Biomatériaux (Chester, Grande-Bretagne, mars 1986) : "un matériau non vivant utilisé et conçu pour interagir avec des systèmes biologiques ». Les biomatériaux peuvent être regroupés en quatre grandes catégories : métaux, céramiques, polymères et matériaux naturels.

Pour un biomatériau, deux types de propriétés sont particulièrement importantes: ses propriétés de volume qui lui confèrent sa biofonctionnalité, c'est-à-dire ses propriétés mécaniques, sa capacité à réaliser une fonction en remplacement de tout ou partie d'un organe (exemple : une prothèse de hanche en titane), mais également ses propriétés de surface qui lui confèrent sa biocompatibilité et éventuellement sa bioactivité. En effet, la surface du matériau est aussi l'interface avec le milieu biologique environnant, constitué de tissus, fluides, cellules et protéines. C'est donc précisément à cette interface que peuvent se jouer des événements d'importance cruciale tels que l'adhésion (ou la non-adhésion) cellulaire, l'activation de macrophages, l'infection bactérienne et la formation de biofilms ${ }^{(1)}$ bactériens. Ainsi, pouvoir modifier les propriétés de surface d'un biomatériau pour lui conférer une activité biologique spécifique est un objectif majeur, sur lequel les chercheurs et les industriels travaillent depuis plusieurs années.

\section{Matériaux pour des applications médicales}

Tout d'abord, citons quelques domaines où il apparaît nécessaire de bien contrôler les propriétés de surface des biomatériaux :

- Implantologie osseuse : le titane est un matériau très utilisé dans la fabrication d'implants osseux tels que les prothèses de hanche, les vis et plaques pour la reconstruction osseuse. II est caractérisé par sa bonne résistance mécanique, sa tenue à la corrosion, sa biocompatibilité, et par un module élastique relativement faible par rapport à d'autres métaux (110 GPa, l'os ayant un module proche de $20 \mathrm{GPa}$ ). Le bon ancrage de la prothèse au sein de l'os et la reconstitution du tissu osseux peuvent être favorisés par le dépôt de couches spécifiques (greffage de facteurs de croissance, de molécules d'adhésion...)

- Domaine vasculaire : plusieurs types de matériaux peuvent être utilisés pour soutenir ou remplacer des vaisseaux défectueux. Ainsi, les "stents " sont des petites prothèses sous forme de grillage (souvent en nickel-titane ou acier inox) destinées à soutenir un vaisseau défaillant chez des patients atteints de maladies artérielles. Des prothèses vasculaires en polymère (Dacron $®$, poly(éthylène)théréphtalate) sont utili-

1. Ensemble de microorganismes développés sur un support. sées pour réaliser des pontages artériels. Un des grands enjeux est d'arriver à recouvrir ces prothèses d'agents antiprolifératifs (comme le taxol) ou anti-coagulants (comme l'héparine). En effet, la bonne intégration des prothèses est freinée par la prolifération excessive des cellules musculaires lisses (cellules constitutives de la paroi interne des vaisseaux) qui a pour conséquence une augmentation de l'épaisseur de la paroi artérielle au détriment du diamètre libre pour l'écoulement du sang.

- Protection microbienne : l'infection microbienne est source d'un grand nombre de rejets d'implants et de complications post-opératoires. Ceci est particulièrement important dans les milieux septiques, pour des prothèses orales ou laryngales. L'étape initiale de l'infection est toujours liée à la capacité des bactéries à adhérer sur la surface des matériaux. $\mathrm{Si}$, dans les premiers temps, cette adhésion est réversible, elle devient irréversible lors du processus infectieux. En raison de problèmes croissants liés à la résistance bactérienne, des peptides antimicrobiens ont été développés en tant que nouveaux agents thérapeutiques.

Diverses techniques de dépôt de molécules actives en surface ont été développées, que nous n'exposerons pas ici. Leur principe repose sur le couplage chimique ou le dépôt de monocouches contenant la molécule active.

Durant la dernière décennie, une nouvelle technique de dépôt de films minces a été proposée [1,2] : il s'agit du dépôt alterné de polyélectrolytes cationiques et anioniques (figure 1) conduisant à la formation de "films multicouches de polyélectrolytes » (FMP) [3] (voir l'encadré sur les polyélectrolytes). Ces films peuvent être déposés sur tout type de surface, quelles que soient sa forme et sa nature. De plus, leur épaisseur varie en fonction des conditions d'édification et du nombre de couches déposées. Ils peuvent être appliqués aux domaines précédemment cités et notamment contenir : 1) des peptides anti-microbiens ou anti-inflammatoires, 2) des médicaments anti-thrombotiques et judicieusement choisis pour empêcher la prolifération des cellules musculaires lisses.

Un autre domaine d'application des FMP est celui de la nano-encapsulation : lorsque les FMP sont déposés sur un support pouvant être dégradé ultérieurement (billes de polystyrène ou cristaux de carbonate de calcium), on forme des capsules creuses. Des principes actifs peuvent être insérés dans ces capsules pour être ultérieurement relargués. Plusieurs équipes travaillent sur cette application à fort potentiel [4].

\section{Fonctionnalisation et multi-fonctionnalisation des films multicouches de polyélectrolytes (FMP)}

Les FMP permettent de créer un « réservoir » de molécules actives en surface du matériau ou d'assurer plusieurs fonc- 


\section{Rappels sur les polyélectrolytes}

- Polyélectrolyte : polymère portant des groupes ionisables, qui peut se dissocier dans un solvant polaire (généralement l'eau) en des chaînes de polymères chargées (macro-ions) et en de petits " contre-ions ". Des exemples typiques de polyélectrolytes naturels sont les protéines, les polysaccharides et l'ADN.

- Polyacides et polybases sont des polymères dont le monomère peut se dissocier et acquérir une charge, en fonction du pH de la solution. Par exemple : la dissociation d'un ion $\mathrm{H}^{+}$d'un polyacide donne lieu à l'apparition d'un groupe $\mathrm{COO}^{-}$et donc d'une charge négative ; la fixation d'un ion $\mathrm{H}^{+}$d'une polybase donne lieu à l'apparition d'un groupe $\mathrm{NH}_{3}{ }^{+}$ et donc d'une charge positive.

Les polyélectrolytes peuvent être forts ou faibles, selon qu'ils sont totalement ionisés ou non en solution. Leur conformation dépend de leur architecture, de leur affinité pour le solvant et de leur charge ; les contreions ont un rôle extrêmement important.

À basse force ionique, les charges tendent à se repousser et la molécule adopte une conformation plus étendue, un peu comme un long bâtonnet (figure A). À forte concentration en sel, les charges ont été écrantées et la chaîne va pouvoir se replier (figure B). La longueur de persistance $L_{p}$ est définie comme étant la longueur sur laquelle la chaîne est rigide (figure $\mathrm{C}$ ).

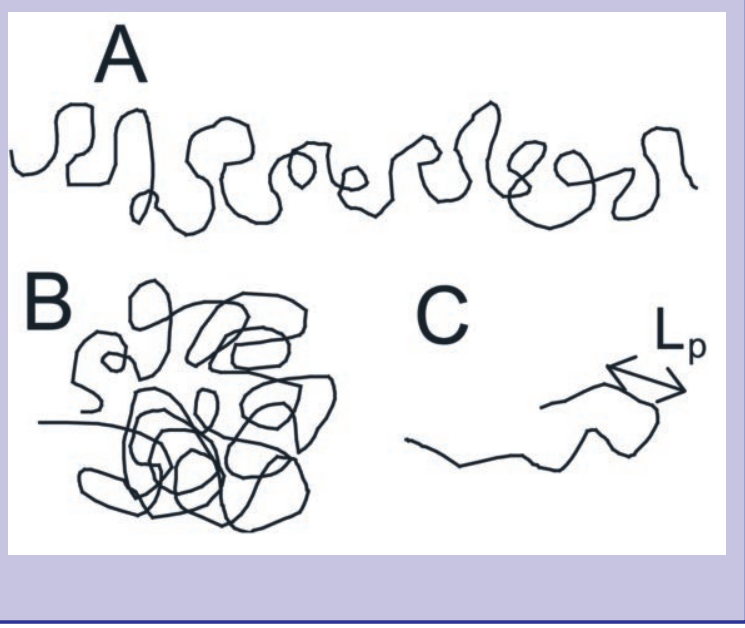

tions par greffage de plusieurs peptides, afin de contrôler la réaction tissulaire et cellulaire au contact du biomatériau. Dans le domaine des biomatériaux, la «fonctionnalisation » désigne le fait de donner une activité biologique à la surface du matériau. Très couramment, une telle fonctionnalisation est réalisée par greffage de molécules directement sur la surface du matériau, mais cette opération peut avoir deux inconvénients : dénaturer la molécule lors du greffage et nécessiter des étapes chimiques complexes à réaliser directement sur le matériau. Les FMP offrent d'une part la possibilité de simplifier cette fonctionnalisation et d'autre part la possibilité de procéder à une multi-fonctionnalisation [5].

Deux méthodes de fonctionnalisation ont été jusqu'à présent adoptées (figure 1) :

- la première consiste à insérer des protéines ou peptides directement, c'est-à-dire par simple adsorption à une étape donnée dans le dépôt des couches. Par exemple, une protéine nommée " protéine $A$ » (protéine pouvant se lier aux immunoglobulines et activer les macrophages) a été récemment insérée dans les FMP pour déclencher la réponse de cellules de type macrophages (réponse inflammatoire). Des immunoglobulines peuvent également être insérées dans les FMP et conduire à une réponse de type antigène-anticorps. Par ce procédé, il est donc possible de déposer à plusieurs reprises des couches de protéines.

- la deuxième méthode consiste à coupler chimiquement une molécule (éventuellement hydrolysable ultérieurement) de type peptide, médicament, ou agent anti-tumoral sur un des poly-
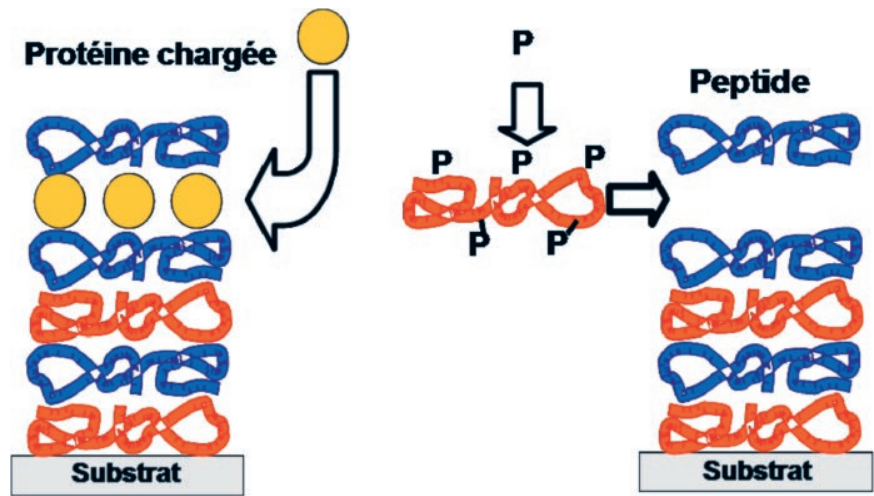

Figure 1. Un FMP est obtenu par dépôt couche par couche de polycations et polyanions. La fonctionnalisation de ce FMP peut consister à enfouir une protéine (figure de gauche), ou à greffer un peptide à l'un des polyélectrolytes puis à adsorber ce dernier sur une couche déjà déposée (figure de droite). électrolytes qui est utilisé pour la construction du FMP. Le greffage du peptide sur le polyélectrolyte est réalisé ex situ, c'està-dire que toutes les étapes chimiques nécessaires au couplage sont réalisées avant l'introduction dans ou sur le film de la molécule couplée. Ceci a notamment pour avantage de ne perturber ni la structure du FMP déposé ni la surface du matériau.

\section{Vers la mise au point de films biomimétiques incluant des polymères naturels ou visant à reproduire les propriétés mécaniques d'un tissu}

Depuis les premiers travaux sur les FMP qui datent du début des années 90, les types de polyélectrolytes utilisés pour fabriquer ces films se sont largement diversifiés. En effet, selon les applications recherchées, il peut être intéressant d'employer des polyélectrolytes synthétiques, des polypeptides, des polysaccharides ou des biopolymères. Dans le domaine biomédical, il faut faire particulièrement attention aux matériaux utilisés quand ils sont implantés dans le corps. La présence d'enzymes, de cellules ayant le rôle de s'attaquer aux corps étrangers (macrophages), les variations de $\mathrm{pH}$, sont autant de facteurs pouvant conduire à la dégradation du film au cours du temps et au relargage de ses constituants. Par conséquent, les polyélectrolytes destinés à des utilisations in vivo doivent être biocompatibles, non toxiques et ne pas générer de réaction inflammatoire trop prononcée.

Ainsi, un choix logique est celui de polyélectrolytes contenus dans les matrices extracellulaires, c'est-à-dire des biopolymères, polysaccharides et protéines constituant la « colle » dans laquelle se trouvent les cellules (cette matrice dépendant de la nature du tissu). Parmi les constituants les plus souvent rencontrés, on trouve le collagène, le hyaluronane (jouant un rôle dans les propriétés mécaniques des tissus et capable de retenir l'eau) et le chitosane (dans la coque des crustacés). Ces molécules sont déjà largement utilisées pour fabriquer des matrices reconstituées, comme substitut de tissu, ou pour mettre au point des systèmes de relargage de médicaments. Leurs avantages sont non seulement leur biocompatibilité et leur biodégradabilité par des enzymes présentes dans les tissus, telles la collagénase pour le collagène ou la hyaluronidase pour le hyaluronane, mais également la possibilité de coupler des molécules actives à ces biopolymères. Ainsi, on peut concevoir que la biodégradabilité des FMP élaborés à base de constituants naturels présentera un intérêt si l'on veut s'en servir comme système de relargage de médicaments en surface d'un matériau. 
En outre, il a été récemment mis en évidence que les propriétés mécaniques des FMP, et en particulier leur rigidité, jouaient un rôle primordial dans l'adhésion cellulaire. En ajustant judicieusement la longueur de persistance des polyélectrolytes (cf. encadré) et en modulant la réticulation des films par une méthode chimique (basée sur la chimie des carbodiimides) applicable en milieu aqueux, il apparait donc possible de mettre au point des films ayant des propriétés mécaniques connues et variables. Notamment, reproduire les propriétés mécaniques d'un tissu au niveau de l'interface matériau/tissu biologique présente un intérêt certain [6]. L'insertion de nanoparticules au sein des FMP est une alternative physique à la rigidification chimique par réticulation ${ }^{(2)}$.

Dans ce cadre, l'équipe du Professeur Kotov [7] a mis au point des films composites à base du polycation chlorure de poly(diallyldimethylammonium) (PDDA) et de tablettes d'argiles montmorillonites chargées négativement. Ces films ont une rigidité similaire à celle de la nacre. Au sein de notre équipe, nous avons réussi à préparer des films à base de hyaluronane ayant un module d'Young entre 5 et $450 \mathrm{kPa}$ [8], ce qui est de l'ordre de grandeur de certains tissus ou gels biologiques (veines, gels de collagènes).

\section{Contrôle de la structure et du dépôt des films}

La cohésion des FMP est assurée par plusieurs types de forces physiques qui dépendent des polyélectrolytes considérés et des conditions d'édification. Les forces électrostatiques sont le principal type de force mis en jeu, l'attraction entre polyélectrolytes de charges opposées permettant l'adsorption d'une couche après l'autre. II est à noter que l'on trouve dans la nature plus de polyanions (hyaluronane, héparine, chondroïtine sulfate, ADN...) que de polycations, le chitosane faisant figure d'exception. Ce polyélectrolyte faiblement chargé est positif à $\mathrm{pH}$ acide. La poly(Lysine) (en forme $\mathrm{L}$ ou $\mathrm{D}$ ) est un autre polycation, synthétisé à partir de l'acide aminé Lysine (cationique). Elle est déjà largement utilisée dans les domaines de la transfection ${ }^{(3)}$ et pour accroître l'adhésion cellulaire sur un substrat.

Les forces hydrophobes semblent également avoir un rôle important. Des films peuvent être construits et stabilisés par des liaisons hydrogène, notamment ceux à base de polyacrylamide à $\mathrm{pH}$ très bas $(\sim 2-3)$.

Quant à l'épaisseur des films, c'est un paramètre contrôlable, non seulement par le nombre de couches déposées, mais également par les conditions de forces ioniques et de $\mathrm{pH}$ qui influencent l'ionisation des polyélectrolytes et leur conformation repliée ou étendue. Les premiers travaux ont concerné des films dont l'épaisseur varie linéairement avec le nombre de couches et se situe dans la gamme du nanomètre à la dizaine de nanomètres.

Plus récemment, un second type de film a été mis au point : des films dont l'épaisseur varie exponentiellement avec le nombre de dépôts [9]. Deux explications ont été proposées pour cette croissance accélérée : la première repose sur une augmentation de la rugosité du film au fur et à mesure de la construction (liée à une augmentation de la surface disponible pour l'adsorption des molécules de la couche suivante) ; une seconde possibilité est la diffusion des polyélectrolytes (un des deux types ou les deux) vers l'intérieur du film lors de leur adsorption et vers l'extérieur lors de l'adsorption du polyélectrolyte de charge opposée [9]. Ainsi, l'épaisseur de tels films, essentiellement à base de polypeptides et polysaccharides, peut atteindre un micromètre après dépôt d'une dizaine de paires de couches. Ces films ont plutôt des propriétés de type « microgels ». L'avantage de ces derniers est que l'on peut y

2. Formation de liaisons covalentes au sein du film.

3. Introduction de matériel génétique étranger dans une cellule. incorporer des peptides ou médicaments, c'est-à-dire qu'ils servent de «réservoir » pour un relargage de produits localisés en surface du matériau. Ce type de film peut également être utilisé comme membrane de filtration. Leur épaisseur relativement grande permet leur observation par microscopie confocale à balayage laser, après marquage fluorescent de l'un des polyélectrolytes, et permet également d'étudier optiquement l'adhésion des cellules en surface du film (figure 2).

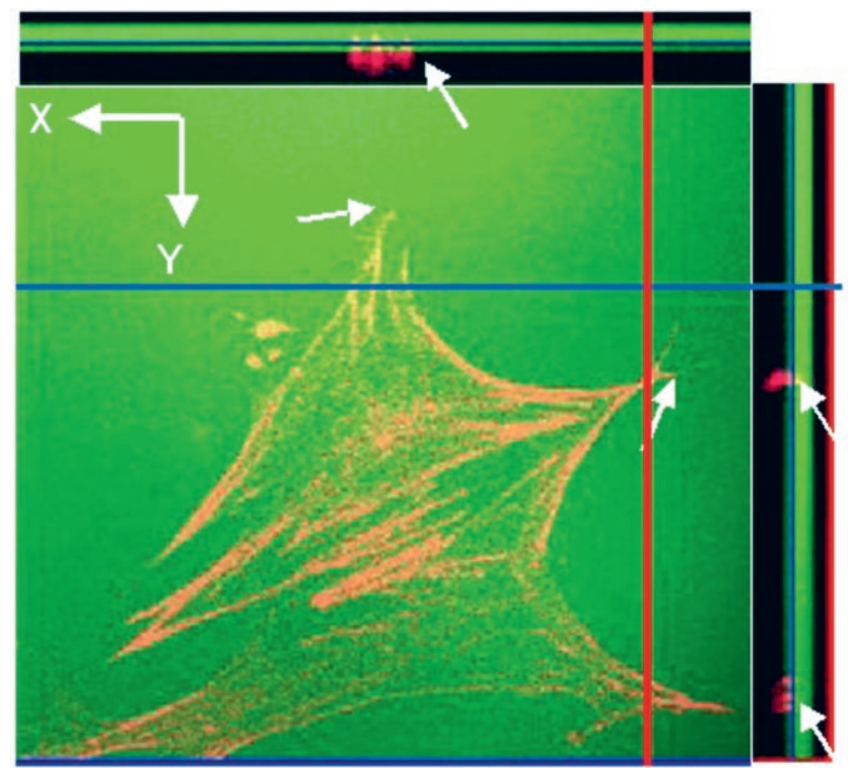

Figure 2. Observation par microscopie confocale de l'adhésion de chondrocytes primaires sur des films (PLL/HA) ${ }_{24}-P L L^{F I T C}$ réticulés (la poly-L-lysine étant couplée à la sonde fluorescente FITC, PLLFITC). Les films sont marqués en vert et la protéine actine du cytosquelette des cellules en rouge. L'image principale représente une coupe au sein d'une cellule (plan $X-Y$ ). Les images supérieure et lattérale montrent respectivement des coupes réalisées en $X-Z$ et en $Y$-Z selon les lignes bleue et rouge présentes sur l'image. Les points de contact entre la cellule et le film apparaissent en jaune (co-localisation verte et rouge). Les flèches blanches indiquent quelques points d'ancrage cellulaire. La taille des images des sections en $Z$ est de $115 \mu \mathrm{m} \times 115 \mu \mathrm{m}$ en $X-Y$; épaisseur du film $\approx 5 \mu \mathrm{m}$.

Enfin, l'utilisation de FMP est envisageable sur différents types de substrats, ainsi que l'ont démontré des travaux récents [10]. Des polymères, métaux et céramiques peuvent être aisément recouverts et leurs propriétés de surface peuvent être considérablement modifiées. II est même envisageable de recouvrir des matrices poreuses afin de favoriser une colonisation cellulaire de ces matrices.

\section{Premiers tests de stabilité et de biodégradabilité des films in vivo}

Récemment, les premiers travaux in vivo concernant les FMP ont été effectués. En effet, les matériaux introduits in vivo ne sont pas soumis aux mêmes sollicitations en fonction des tissus ou des fluides qui sont à leur contact. Chaque étude doit être réalisée dans des conditions spécifiques, qui correspondent à la localisation de la future implantation. L'équipe du Professeur Debry (CHU de Strasbourg) réalise actuellement des implantations de prothèses trachéales en titane fritté recouvertes de films multicouches dans lesquels un agent anti-inflammatoire a été inséré. Avec le Dr Etienne de la Faculté de Chirurgie Dentaire, nous avons réalisé une implantation en milieu buccal de disques polymériques recouverts de films à base de polysaccharides et avons étudié la biodégradabilité de films non réticulés et de films réticulés (par la méthode chimique précédemment citée) sur une période de six heures à trois jours (figure 3).

Nous avons également étudié in vitro le contact avec des enzymes salivaires (lysozyme, amylase) et des bactéries, en 

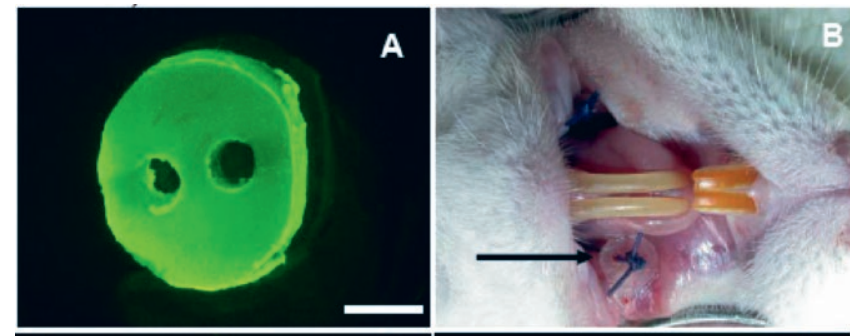

C

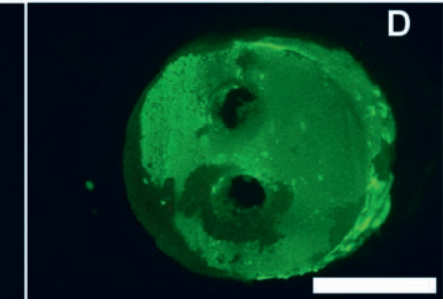

Figure 3. Expériences réalisées in vivo dans la cavité orale de rats. Des disques en PMMA (poly(méthyl méthacrylate) de diamètre $4 \mathrm{~mm}$ ont été recouverts sur les deux faces d'un film (CHI/HA) ${ }_{24}-\mathrm{CHI}$ FITC à l'aide d'un bras automatisé (l'épaisseur du film est de 6 um environ) (film contrôle, A) et suturés dans la bouche de rats par l'intermédiaire de deux trous percés dans les disques (B). Les images d'un film natif $(C)$ et d'un film réticulé (D) ont été prises après trois jours d'implantation dans la cavité orale des rats (barre d'échelle $2 \mathrm{~mm}$, taille des images $6,4 \times 4,8 \mathrm{~mm}^{2}$ pour $A, C$ et D). Les films natifs sont très rapidement dégradés et seules quelques traces de films sont visibles après trois jours (l'aspect rouge de l'image provient de l'auto-fluorescence de la résine), alors que les films réticulés résistent beaucoup mieux. En effet, la fluorescence est observable sur la quasi-totalité du film, hormis quelques parties dégradées (notamment sur les bords et autour des trous centraux).

milieu plutôt acide. Pour une implantation vasculaire, il faut en revanche étudier le contact avec le sang et les cellules de type macrophage. C'est pourquoi nous avons introduit ces mêmes films dans la poche péritonéale de la souris, afin d'examiner la réponse inflammatoire due aux macrophages (sur une durée allant jusqu'à 21 jours). II apparaît que les films réticulés sont plus résistants que les films non réticulés et que l'adhésion des macrophages dépend du taux de réticulation des films [11].

\section{Conclusions et perspectives}

Dans le domaine du traitement de surface des biomatériaux, il est apparu au cours des dernières années que les propriétés mécaniques du support sont extrêmement importantes pour l'adhésion des cellules. Jusqu'alors, l'influence des propriétés chimiques de la surface (groupements fonctionnels, hydrophilie...) et de la topographie de la surface (microgravures, rugosités...) avaient surtout été étudiées. II paraît désormais important d'élucider plus avant le rôle des propriétés mécaniques sur le comportement cellulaire (prolifération, différentiation), dans le but de fabriquer des recouvrements aux propriétés mécaniques biomimétiques, c'est-àdire aussi proches que possible de celles de la paroi artérielle, de l'os, des tendons...

En ce qui concerne plus spécifiquement les FMP, il faut tout d'abord poursuivre les études fondamentales destinées à mieux comprendre et contrôler leurs propriétés structurales, mécaniques, l'insertion de molécules actives notamment d'ADN ou de facteurs de croissance spécifiques pour favoriser la différentiation de certains types cellulaires, leur éventuel relargage (ce qui fait appel à des notions de diffusion en milieu confiné).

Le comportement des cellules au contact des films et notamment l'adhésion, la prolifération et la différentiation cellulaire doit être mieux compris et contrôlé. Ces films représentent une formidable opportunité d'étudier des mécanismes fondamentaux d'adhésion, grâce au grand nombre de paramètres contrôlables et à une gamme d'épaisseurs (de quelques nanomètres à quelques centaines de nanomètres voire micromètres) qui est difficilement accessible par d'autres méthodes de dépôt. De plus, des techniques microscopiques et spectroscopiques performantes, telles que les microscopies à force atomique et de fluorescence à réflexion totale interne, des spectroscopies à onde évanescente ou plasmon de surface sont parfaitement adaptées à l'étude de ces films minces et permettront à la fois de mieux comprendre la structure des films et leur interaction avec des cellules.

Les études in vivo qui ont débuté avec les cliniciens vont très certainement prendre de l'ampleur et être plus spécialisées, en fonction du type d'application recherché. Un grand enjeu est notamment de valider le concept de fonctionnalisation des films in vivo en mettant au point des tests de fonctionnalité.

Dans ce domaine, comme dans beaucoup d'autres, c'est en rassemblant des physiciens, chimistes, biologistes et cliniciens qui travaillent ensemble sur de multiples aspects d'un même matériau, que nous arriverons à innover et à rester dans la compétition internationale.

\section{Glossaire}

Biofonctionnalité : Capacité d'un matériau à remplir la fonction pour laquelle il a été conçu (par exemple, pour une prothèse de hanche : remplacer une articulation).

Biocompatibilité : Capacité d'un matériau à être utilisé avec une réponse appropriée de l'hôte pour une application spécifique.

Bioactivité : Capacité d'un matériau à induire une réponse biologique positive des cellules ou tissus environnants (par exemple expression de protéines, différentiation des cellules...).

Chondrocyte : Cellule du cartilage.

Macrophage : Cellule du système immunitaire, dont le rôle est de phagocyter (détruire en absorbant) les corps étrangers.

Cytosquelette (actine) : Charpente de la cellule, constituée notamment de la protéine " actine ", qui forme des filaments dont la longueur de persistance est de $17 \mu \mathrm{m}$.

Polypeptide : polymère d'acides aminés; les acides aminés, au nombre de 20 , sont l'unité de base de toutes les protéines ; ils comportent une fonction acide carboxylique et une fonction amine.

Polysaccharide : polymère de saccharide ; les saccharides sont des oses (molécules cycliques de formule $\mathrm{C}_{n}\left(\mathrm{H}_{2} \mathrm{O}\right)_{n}, \mathrm{n}=3$ à 7 ).

Collagène : principale protéine constitutive des tissus biologiques, se trouvant sous plus de dix formes différentes dans la nature.

Hyaluronane : polymère d'une unité disaccharide dont la longueur totale peut varier en fonction du nombre d'unités répétitives (typiquement de 100 à 3000). On le trouve dans un grand nombre de tissus et fluides tels que la peau, le cartilage, le fluide synovial, I'humeur vitreuse.

\section{Remerciements}

C. Picart remercie Jean-Claude Voegel (INSERM UMR595), Pierre Schaaf et Gero Decher (Institut Charles Sadron, Strasbourg) pour les nombreuses discussions scientifiques.

\section{Références}

[1] Decher, G., Science, 277 (1997), 1232-1237.

[2] Schaaf, P. et al., La Recherche, 389 (2005), 56-58

[3] Barrat, J. et Joanny, J.F., Advances in Chemical Physics, XCIV (1996), 1-66.

[4] Antipov, A.A. et Sukhorukov, G.B., Adv. Colloid Interface Sci., 111 (2004), 49-61.

[5] Lvov, Y.M. in Handbook of Surfaces and Interfaces of Materials ; Vol 3: Nanostructured Materials, Micelles and Colloids; Nalwa, H. S., Ed.; Academic Press, 2001.

[6] Chevallard, C. et Guénoun, P., Bulletin de la SFP, 155 (2006), pp. 5-10

[7] Tang, Z. et al., Nat. Mater., 2 (2003), 413-418.

[8] Schneider, A. et al., Langmuir, 22 (2006), 1193-1200.

[9] Picart, C. et al., Proc. Natl. Acad. Sci., 99 (2002), 12531-12535.

[10] Tan, Q. et al., Biomaterials, 24 (2003), 4699-4705.

[11] Picart, C. et al., Adv. Funct. Mater., 15 (2005), 1771-1780. 